P. Jothilingam

Nagoya Math. J.

Vol. 59 (1975), 149-152

\title{
A NOTE ON GRADE
}

\section{P. JOTHILINGAM}

All rings that occur in this note will be assumed to be commutative with unity and all modules will be finitely generated and unitary.

The grade of a module $M$ over a noetherian local ring $R$ is defined to be the length of a maximal $R$-sequence contained in the annihilator of $M$. If $M$ has finite projective dimension it is well-known that grade $M \leq$ proj. $\operatorname{dim} M$. We can say more when $R$ is a regular local ring. We state the

THEOREM. Let $R$ be a regular local ring and $M$ a given $R$-module. Let $N$ be any other $R$-module such that $\operatorname{Hom}(M, N) \neq(0)$. Let $p$ be the least integer such that $\operatorname{Ext}_{R}^{p}(M, N)=(0)$. Then grade $M \leq \inf (p-1$, proj. $\operatorname{dim} N)$. If $q$ is the least integer such that $\operatorname{Ext}_{R}^{q}(M, M)=(0)$, then projective dimension of $M$ equals $q-1$.

Remark. Taking $N=k$, we get grade $M \leq$ proj. $\operatorname{dim} M$, the result mentioned in the introduction.

The proof of theorem depends on the following

LEMma. Let $R$ be a regular local ring; let $M, N$ be any two $R$ modules. If $\operatorname{Ext}_{R}^{p}(M, N)=(0)$ for some integer $p \geq 1$, then there exists a natural isomorphism $\operatorname{Ext}_{R}^{p-1}(M, R) \bigotimes_{R} N \cong \operatorname{Ext}_{R}^{p-1}(M, N)$.

Proof. Define $\Omega^{0}=M$ and for $p \geq 1$, define $\Omega^{p}$ to be the $p$ th syzygy module of $M$ taken with respect to a fixed minimal resolution of $M$,

$$
\rightarrow F_{n} \rightarrow F_{n-1} \rightarrow \cdots \rightarrow F_{0} \rightarrow M \rightarrow 0
$$

Taking the $R$-dual sequence of (1) and using * to denote $R$-duals we define $D \Omega^{p}=\operatorname{cokernel}\left(F_{p}^{*} \rightarrow F_{p+1}^{*}\right)$ for $p \geq 0$. According to [3] for every integer $p \geq 0$, there exists an exact sequence

$$
\operatorname{Tor}_{2}^{R}\left(D \Omega^{p}, N\right) \rightarrow \operatorname{Ext}_{R}^{p}(M, R) \otimes N \rightarrow \operatorname{Ext}_{R}^{p}(M, N) \rightarrow \operatorname{Tor}_{1}^{R}\left(D \Omega^{p}, N\right) \rightarrow 0
$$

Received December 19, 1974. 


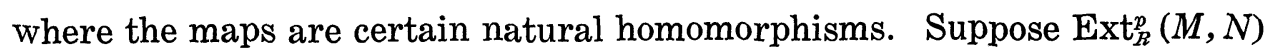
is the zero module. Applying (2) we obtain $\operatorname{Tor}_{1}^{R}\left(D \Omega^{p}, N\right)=(0)$. Using [6] this implies $\operatorname{Tor}_{j}^{R}\left(D \Omega^{p}, N\right)=(0)$ for $j \geq 1$. The second application of (2) yields $\operatorname{Ext}_{R}^{p}(M, R) \otimes N=(0)$. Since $N \neq(0)$, we conclude that $\operatorname{Ext}_{R}^{p}(M, R)=(0)$, i.e. $\operatorname{Ext}_{R}^{1}\left(\Omega^{p-1}, R\right)=(0)$. Taking $R$-duals in the exact sequence $0 \rightarrow \Omega^{p} \rightarrow F_{p-1} \rightarrow \Omega^{p-1} \rightarrow 0$ and using the fact that $\operatorname{Ext}_{R}^{1}\left(\Omega^{p-1}, R\right)$ $=(0)$, we obtain the following exact sequence

$$
0 \rightarrow\left(\Omega^{p-1}\right)^{*} \rightarrow F_{p-1}^{*} \rightarrow\left(\Omega^{p}\right)^{*} \rightarrow 0
$$

Using the definition of $D \Omega^{p}$, we get an exact sequence

$$
0 \rightarrow\left(\Omega^{p}\right)^{*} \rightarrow F_{p}^{*} \rightarrow F_{p+1}^{*} \rightarrow D \Omega^{p} \rightarrow 0
$$

Putting (2) and (3) together and making use of the definition of $D \Omega^{p-1}$, we get the following exact sequence,

$$
0 \rightarrow D \Omega^{p-1} \rightarrow F_{p+1}^{*} \rightarrow D \Omega^{p} \rightarrow 0 .
$$

The exact sequence (5) gives $\operatorname{Tor}_{j}^{R}\left(D \Omega^{p-1}, N\right)=\operatorname{Tor}_{j+1}^{R}\left(D \Omega^{p}, N\right)=(0)$ for $j \geq 1$. The lemma follows after using this information in the exact sequence (2) with $p$ replaced by $p-1$.

Proof of the theorem: If $\operatorname{grade} M>\operatorname{proj} . \operatorname{dim} N$, then clearly $\operatorname{depth} N>\operatorname{Krull} \operatorname{dim} M$ and so applying [4] we find that $\operatorname{Hom}(M, N)=(0)$, a contradiction. Hence grade $M \leq$ proj. $\operatorname{dim} N$. The lemma gives an isomorphism $\operatorname{Ext}_{R}^{p-1}(M, R) \otimes N \cong \operatorname{Ext}_{R}^{p-1}(M, N)$. Now if grade $M \geq p$, it is well-known that $\operatorname{Ext}_{R}^{i}(M, R)=(0)$ for $0 \leq i \leq p-1$, so that $\operatorname{Ext}_{R}^{p-1}(M$, $N)=(0)$, a contradiction to the minimality of $p$. Hence grade $M \leq p-1$. Combining with the inequality grade $M \leq$ proj. $\operatorname{dim} N$ established before we find that grade $M \leq \inf (p-1$, proj. $\operatorname{dim} N)$. This proves the first part of the theorem. As for the second part we observe that $\operatorname{Ext}_{R}^{q}(M$, $M)=(0)$ implies, as in the lemma above that $\operatorname{Tor}_{j}^{R}\left(D \Omega^{q-1}, M\right)=(0)$ for $j \geq 1$. Using this in the exact sequence $0 \rightarrow \Omega^{q-1} \rightarrow F_{q_{-2}} \rightarrow \cdots \rightarrow F_{0} \rightarrow$ $M \rightarrow 0$ we get $\operatorname{Tor}_{j}^{R}\left(D \Omega^{q-1}, \Omega^{q-1}\right)=(0)$ for $j \geq 1$. An application of (2) with $p=0$ and $M$ replaced by $\Omega^{q-1}$ shows that the natural map $\left(\Omega^{q-1}\right)^{*}$ $\otimes \Omega^{q-1} \rightarrow \operatorname{Hom}\left(\Omega^{q-1}, \Omega^{q-1}\right)$ is an isomorphism. Hence $\Omega^{q-1}$ is projective, i.e. proj. $\operatorname{dim} M \leq q-1$. The minimality of $q$ implies that proj. $\operatorname{dim}$ $M=q-1$.

The theorem is proved. 
We recall the following conjecture of M. Auslander

TOR CONJECTURE: If $M$ is a module of finite projective dimension over a noetherian local ring $R$ and $N$ any other $R$-module such that

$$
\operatorname{Tor}_{1}^{R}(M, N)=(0), \text { then } \operatorname{Tor}_{j}^{R}(M, N)=(0) \text { for } j \geq 1 .
$$

It is well-known that this conjecture is true if $R$ is regular local [6] and trivially so if proj. $\operatorname{dim} M \leq 1$. We remark that if the above conjecture is true then the lemma is valid for any noetherian local ring provided $N$ has finite projective dimension. Consequently the second part of the theorem is also valid for any noetherian local ring provided $M$ has finite projective dimension.

M. Auslander and $O$. Goldman have proved that a reflexive module $M$ over a regular local ring $R$ is free if and only if $\operatorname{Hom}(M, M)$ is free [1]. In his article on the purity of the Branch locus [2] M. Auslander asks if this result is true for any noetherian local ring provided one assumes that $M$ has finite projective dimension. We shall show that the answer is yes if the Tor conjecture mentioned above is true. In fact we prove the following

Propositition. Let $M, N$ be reflexive modules of finite projective dimensions over a noetherian local ring $R$ such that $\operatorname{Hom}(M, N)$ is a nonzero free $R$-module. Then if the Tor conjecture is true $M$ and $N$ are both free modules.

Proof. By induction on the Krull-dimension of $R$ and [1, Lemma 4.8] we easily find that $\operatorname{Ext}_{R}^{1}(M, N)=(0)$. As in the proof of the lemma we get an isomorphism $M^{*} \otimes N \cong \operatorname{Hom}(M, N)$. Hence $M^{*} \otimes N$ is a nonzero free module. From this it is easy to conclude that both $M^{*}$ and $N$ are free. Since $M$ is reflexive, $M$ and $N$ are both free modules.

\section{REFERENCES}

[1] M. Auslander and O. Goldman: Maximal order, Trans. Amer. Math. Soc. Vol. 97 (1960).

[2] M. Auslander: On the purity of the branch locus, American Journal of Mathematics Vol. 84 (1962).

[3] M. Auslander: Coherent functors, Proceedings of a conference on categorical algebra, La Jolla 1965, Springer-Verlag Berlin, Heidelberg, New York 1966.

[4] F. Ischebeck: Eine Dualitat zwischen den Funktoren Ext und Tor, J. Algebra 11 (1969), 510-531.

[ 5 ] D. Rees: The grade of an ideal or module, Proc. Camb. Phil. Soc. 53 (1957). 
[6] S. Lichtenbaum: On the vanishing of Tor in regular local rings Illinois. J. of Math. Vol. 10 (1966).

Tata Institute of Fundamental Research 